\section{Evaluation of basal cortisol testing in dogs with chronic gastrointestinal signs}

\section{Ana Fernandez Gallego, Alisdair Boag, Adam Gow}

Hospital for Small Animals, Royal (Dick) School of Veterinary Studies, Edinburgh, United Kingdom

\section{OBJECTIVES}

Basal cortisol concentration $<55 \mathrm{mmol} / \mathrm{L}$, a screening test for hypoadrenocorticism, was evaluated in dogs with chronic gastrointestinal signs presenting to a referral Internal Medicine Service.

\section{METHODS}

All dogs with chronic gastrointestinal signs (vomiting, diarrhoea, weight loss, stunted growth, or a combination, for 3 weeks or longer) had basal cortisol performed between May 2013 and September 2017. Records were reviewed, for signalment, $\mathrm{ACTH}$ stimulation results, and final diagnosis.

\section{RESULTS}

Of 298 cases, basal cortisol was $<55 \mathrm{mmol} / \mathrm{L}$ in 88 (29.53\%). An ACTH stimulation test was performed in all cases with cortisol $<13.8 \mathrm{mmol} / \mathrm{L}(8)$, and in $57 / 80$ with cortisol between $13.8-55 \mathrm{mmol} / \mathrm{L}$. Hypoadrenocorticism was the final diagnosis in one dog $(0.33 \%)$. In patients with cortisol $<55$ $\mathrm{mmol} / \mathrm{L}$ where an ACTH stimulation test was not performed (23/80), the most common diagnosis was chronic primary inflammatory enteropathy (13), a final diagnosis was not reached in 2 of these patients. Overall, the most common diagnosis was chronic primary inflammatory enteropathy (58.72\%), followed by protein-losing enteropathy $(6.04 \%)$, then megaoesophagus and pancreatitis (3.69\% each).

\section{STATEMENT (CONCLUSIONS)}

Basal cortisol has not been previously assessed specifically in a population presenting to a referral Internal Medicine Service with chronic gastrointestinal signs. Hypoadrenocorticism can manifest with chronic gastrointestinal signs; however, it was the final diagnosis in only $1 / 298$ dogs and only $1 / 88$ with basal cortisol $<55 \mathrm{mmol} / \mathrm{L}$. Primary inflammatory enteropathy was the final diagnosis in more than half of the patients, both with basal cortisol lower and greater than $55 \mathrm{mmol} / \mathrm{L}$.

\section{Co-morbidities associated with diabetes mellitus in Burmese cats}

\section{Andrew Fisher, Rebecca Geddes, Katarina Hazuchova}

The Royal Veterinary College, London, United Kingdom

\section{OBJECTIVES}

Diabetes mellitus (DM) is a common feline endocrinopathy and Burmese cats are predisposed. This breed has limited genetic diversity, but not all Burmese cats develop DM, suggesting there are other factors associated. This study aimed to ascertain which co-morbidities are associated with DM in Burmese cats.

\section{METHODS}

An online survey was developed to collect data regarding signalment, previous or current medications and morbidities of Burmese cats. Owners were invited to complete the questionnaire for all Burmese cats. Cats were categorised into DM and non-diabetic (nDM) groups. Proportions of animals affected with each morbidity between groups were analysed using Chi-squared or Fisher's exact tests. Mann-Whitney $U$ tests were used to compared non-parametric continuous data between groups.

\section{RESULTS}

DM cats were significantly older than nDM cats and no DM cat was $<10$ years of age. nDM cats $<10$ years were subsequently excluded. Comparisons between agematched DM and nDM groups found DM to be significantly associated with obesity (OR 7.86, 95\% Cl 2.82-21.03), hypokalaemia (OR 27.47, 95\% Cl 3.873317.60 ), cystitis or urinary tract infection (OR $2.78,95 \%$ Cl 1.09-7.21), pancreatitis (OR 4.16, 95\% Cl 1.30-12.24), dental disease (OR 2.50, 95\% Cl 0.97-6.46), and anaemia (OR Infinity, 95\% Cl 1.67- ¥).

\section{STATEMENT (CONCLUSIONS)}

Vets should be aware that Burmese cats with these conditions may be at increased risk of DM and/or DM Burmese should be evaluated for development of these co-morbidities. Prospective studies to investigate causal relationships between DM and these co-morbidities are sought after. 